BULL. AUSTRAL. MATH. SOC.

VOL. I (1969), 307-314

\title{
Some arithmetical identities for Ramanujan's and divisor functions
}

\author{
D. B. Lahiri
}

A new linear expression in $\sigma(\nu), \nu=1,2, \ldots, n$, which vanishes identically is established. A linear expression in $\sigma(\nu)$ 's has been found for $\sigma_{3}(n)$. A similar expression in $\sigma_{3}(v)$ 's has been proved for $\sigma_{7}(n)$ also. Ramanujan's $\tau(n)=p_{24}(n-1)$ is given in three different ways as linear expressions in $\sigma_{2 k+1}(n)$ and $\sigma_{k}(\nu)$ 's with $k=1,3,5$ respectively. Again, the coefficient $p_{48}(n-2)$ is expressed as a linear expression in $\sigma_{1 l}(\nu)^{\prime} s$ and $\sigma_{5}(\nu)^{\prime} s$. In establishing these results advantage is taken of the general theorem, also established, that the coefficients of the square of a power series whose coefficients satisfy a certain functional equation are expressible as linear functions of the latter coefficients.

\section{Introduction and final results}

There is a beautiful classical identity [7, p. 212] involving the arithmetical function $\sigma(n)$, the sum of the divisors of $n$. This may be stated as

$$
\sum_{v} \pm \sigma(n-v)=0
$$

where the summation is over the pentagonal numbers

$$
v=\frac{1}{2} m(3 m+1), m=0, \pm 1, \pm 2, \pm 3, \ldots \ldots \text {, }
$$

with the understanding that the sign to be prefixed to the term $\sigma(n-v)$ is

Received 21 April 1969. Received by J. Austral. Math. Soc. 12 February 1969. Communicated by E.S. Barnes. 
positive or negative according as

$$
v=(2 m+1)(3 m+1) \text { or } v=m(6 m+1)
$$

respectively, and further $\sigma(0)=n$ and $\sigma(m)=0$ if $m<0$. An interesting problem is to find other identities in which, as is in the relation (1) above, the only arithmetical function involved is $\sigma(\cdot)$ with arguments which are all functions of the integral variable $n$. An answer is provided by the following theorem which will be established later on.

THEOREM 1. $(n-1) \sigma(n)-6 n \sum_{d \mid n}\left[\frac{1}{d^{3}} \sum_{r}^{\prime}\left(d^{2}-5 r^{2}\right) \sigma\left(\frac{d^{2}-r^{2}}{4}\right)\right]=0$.

The arguments in the above theorem unlike those in (1) involve squares instead of pentagonal numbers. Also unlike that in (1), $\sigma(0)=0$. In fact we shall adopt the convention throughout the paper, - with the single exception stated for ( 1 ), - that all terms involving negative, zero or fractional arguments which might appear in a function which is defined strictly for positive integral values only are really vanishing. The sum $\sum_{n}$ extends over the divisors of $n$. We shall use $\sum_{r}$ to denote a sum extended over all positive integers $r$, and $\sum_{r}^{\prime} F(r)$ would be used to mean $\frac{1}{2} F(0)+\sum_{r} F(r)$

Another problem which the author has considered is to express $\sigma_{k}(n)$, the elementary divisor function of degree $k$, in terms of divisor functions of lower degree. The answers for $k=3$ and 7 are shown in the following theorems. For the sake of uniformity we have used $\sum_{r}^{\prime}$ in Theorem 2 even though $\sum_{r}$ could have been used as well.

THEOREM 2. $\sigma_{3}(n)=(2 n-1) \sigma(n)+24 n \sum_{d\rceil_{n}}\left[\frac{1}{d^{3}} \sum_{r}^{1} r^{2} \sigma\left(\frac{d^{2}-r^{2}}{4}\right)\right] ;$

THEOREM 3. $\sigma_{7}(n)=\sigma_{3}(n)+240 n^{3} \sum_{d\rceil n}\left[\frac{1}{d^{3}} \sum_{r}^{1} \sigma_{3}\left(\frac{d^{2}-r^{2}}{4}\right)\right]$.

In connection with Theorems 2 and 3 it is relevant to point out that the author has in an earlier paper [6] given expressions for 
$\sigma_{k}(n), k=1,3,5,7,9$ in terms of an arithmetical function of a different category, namely, the unrestricted partition function $p(n)$. As illustrations we give below the cases corresponding to $k=3$ and 7 .

$$
5 \sigma_{3}(n)=\sum_{v} \pm\left[18 n-d_{1}(v)\right] \cdot v p(n-v)
$$

$$
5 \sigma_{7}(n)=\sum_{v} \pm\left[1512 n^{3}-504 d_{1}(v) \cdot n^{2}+42 d_{2}(v) \cdot n-d_{3}(v)\right] \cdot v p(n-v)
$$

where the polynomials $d_{i}(v), i=1,2,3$ are as follows:

$$
\begin{aligned}
& d_{1}(v)=1+12 v, \\
& d_{2}(v)=1+24 v+192 v^{2}, \\
& d_{3}(v)=1+36 v+576 v^{2}+3456 v^{3} .
\end{aligned}
$$

The meznod followed in establishing the above theorems leads us naturally to expressions for Ramanujan's $\tau(n)$ in terms of $\sigma_{k}(n)$ 's where $\tau(n)$, as is well known, is defined by

$$
x\left[\prod_{n=1}^{\infty}\left(1-x^{n}\right)\right]^{24}=\sum_{n=1}^{\infty} \tau(n) x^{n} .
$$

These expressions are given below.

THEOREM 4. For alZ positive integral values of $n$

$$
\begin{aligned}
\tau(n) & =\frac{15}{8} n^{4} \sigma_{3}(n)-\frac{7}{8}\left(6 n^{5}-5 n^{4}\right) \sigma(n)-105 n^{5} \cdot \sum_{d\rceil n}\left[\frac{1}{d^{5}} \sum_{r}^{\prime} r^{4} \sigma\left(\frac{d^{2}-r^{2}}{4}\right)\right], \\
& =-\frac{1}{8} n^{2} \sigma_{7}(n)+\frac{9}{8} n^{2} \sigma_{3}(n)+270 n^{5} \cdot \sum_{d{ }_{n}}\left[\frac{1}{d^{5}} \sum_{r}^{\prime} r^{2} \sigma_{3}\left(\frac{d^{2}-r^{2}}{4}\right)\right], \\
& =\frac{65}{756} \sigma_{11}(n)+\frac{691}{756} \sigma_{5}(n)-\frac{1382}{3} n^{5} \cdot \sum_{d\rceil n}\left[\frac{1}{d^{5}} \sum_{r}^{\prime} \sigma_{5}\left(\frac{d^{2}-r^{2}}{4}\right)\right] .
\end{aligned}
$$

Incidentally a pair of expressions of a different type are given for $\tau(n)$ in the paper [6]. There not only $\sigma_{k}(n)$ 's are involved as in Theorem 4 but also $p(n)$.

Another function to which the method is applicable is $p_{48}(n)$. The functions $p_{k}(n)$ defined for different values of $k$ by 


$$
\left[\prod_{1}^{\infty}\left(1-x^{n}\right)\right]^{k}=\sum_{0}^{\infty} p_{k}(n) x^{n}
$$

have received considerable attention. Explicit expressions for the values of $p_{k}(n)$ for the cases $k=1$ and 3 are given respectively by the famous identities of Euler and Jacobi. It is of interest to find explicit values of $k$. We have already referred to such expressions for $k=24$, - we recall $p_{24}(n-1)=\tau(n)$. We shall now give for $p_{48}(n)$ a finite series involving the divisor functions only as in the previous theorems, in addition to one involving Ramanujan's function.

THEOREM 5. $p_{48}(n-2)=2 n^{11} \sum_{d\rceil n}\left[\frac{1}{d^{11}} \cdot \sum_{r}^{\prime} \tau\left(\frac{d^{2}-r^{2}}{4}\right)\right]$ $=2 n^{11} \sum_{d\rceil n}\left[d^{-11} \sum_{r}^{\prime}\left\{65 \sigma_{11}(\beta)+691 \sigma_{5}(\beta)-348264 \beta^{5} \cdot \sum_{t\lceil\beta} t^{-5} \sum_{r}^{\prime} \sigma_{5}(\beta)\right\}\right]$ where

$$
\beta=\frac{1}{4}\left(d^{2}-r^{2}\right)
$$

\section{Some lemmas}

LEMMA 1. $D(p n)=D(p) \cdot D(n)-p^{k+2 \alpha} \cdot D(n / p)$ where $p$ is any prime and

$$
D(n)=n^{\alpha} \sigma_{k}(n), \quad \alpha, k \geqq 0 .
$$

The above lemma can be proved from the following identities which can be established successively without any difficulty.

$$
\begin{aligned}
\sigma_{k}\left(p^{\lambda+1}\right) & =\sigma_{k}(p) \cdot \sigma_{k}\left(p^{\lambda}\right)-p^{k} \cdot \sigma_{k}\left(p^{\lambda-1}\right), \lambda \geqq 0, \\
\sigma_{k}(p n) & =\sigma_{k}(p) \cdot \sigma_{k}(n)-p^{k} \cdot \sigma_{k}(n / p), \\
(p n)^{\alpha} \sigma_{k}(p n) & =p^{\alpha} \sigma_{k}(p) \cdot n^{\alpha} \sigma_{k}(n)-p^{k+2 \alpha} \cdot\left(\frac{n}{p}\right)^{\alpha} \sigma_{k}\left(\frac{n}{p}\right) . \\
\text { LEMMA 2. } 12\left[\sum_{-1}^{\infty} \sigma(n) x^{n}\right]^{2} & =\sum_{1}^{\infty}\left[5 \sigma_{3}(n)-(6 n-1) \sigma(n)\right] x^{n}, \\
12\left[\sum_{1}^{\infty} n \sigma(n) x^{n}\right]^{2} & =\sum_{1}^{\infty}\left[n^{2} \sigma_{3}(n)-n^{3} \sigma(n)\right]_{x^{n},}, \\
120\left[\sum_{1}^{\infty} \sigma_{3}(n) x^{n}\right]^{2} & =\sum_{1}^{\infty}\left[\sigma_{7}(n)-\sigma_{3}(n) x_{x} n .\right.
\end{aligned}
$$


The above identities are already known; they are respectively mere restatements of the identities (3.1), (3.3) and (7.1) of Table $B(1)$ given by the author in [4] where we have written

$$
\sum_{1}^{\infty} n^{r} \sigma_{s-r}(n) x^{n}=(r, s)
$$

for the sake of simplicity.

LEMMA 3. $840\left[\left[n_{1}^{\infty} n^{2} \sigma(n) x^{n}\right]^{2}=\sum_{1}^{\infty}\left[15 n^{4} \sigma_{3}(n)-14 n^{5} \sigma(n)-\tau(n)\right] x^{n}\right.$,

$$
\begin{aligned}
540\left[\sum_{1}^{\infty} n \sigma_{3}(n) x^{n}\right]^{2} & =\sum_{1}^{\infty}\left[n^{2} \sigma_{7}(n)-\tau(n)\right] x^{n}, \\
174132\left[\sum_{1}^{\infty} \sigma_{5}(n) x^{n}\right]^{2} & =\sum_{1}^{\infty}\left[65 \sigma_{11}(n)+691 \sigma_{5}(n)-756 \tau(n)\right] x^{n} .
\end{aligned}
$$

The above identities are also known; they are respectively mere restatements of the identities (3.2), (7.1) and (11.1) of Table $B(2)$ given in [5] where we have written

$$
\sum_{1}^{\infty} \tau(n) x^{n}=\{0\}
$$

\section{The basic theorem}

All the theorems stated in the introductory section require for their proof the following basic theorem.

THEOREM 0 . If $N(n)$ is a function of the integral variable $n>0$ such that for any prime $p$

$$
N(p n)=N(p) N(n)-\lambda(p) N(n / p)
$$

where $\lambda(x)$ is completely multiplicative, that is,

$$
\lambda(u v)=\lambda(u) \lambda(v), \lambda(u) \neq 0,
$$

$u$ and $v$ being any arbitrary pair of positive numbers, then

$$
\sum_{l n}\left[\frac{2}{\lambda(d)} \sum_{r}^{\prime} N\left(\frac{d^{2}-r^{2}}{4}\right)\right]=\frac{1}{\lambda(n)} \cdot M(n)
$$

where 


$$
\left[\sum_{1}^{\infty} N(n) x^{n}\right]^{2}=\sum_{1}^{\infty} M(n) x^{n}
$$

The validity of the above theorem can be seen from the following observations. Formulas have been given by Hurwitz [3] for the number of ways a square can be expressed as the sum of 3 and 5 squares. While extending the results to 7 squares [8] and to 9,11 and 13 squares [9], Sandham pointed out that Hurwitz's arguments applies to more general numbers, and he gave in [9] a theorem in three parts. The part (or rather an important particular case of it) which is relevant for our purpose is that the coefficient of $q^{m^{2}}$ in the product

$$
\left(1+2 q^{1^{2}}+2 q^{2}+2 q^{3^{2}}+\ldots \ldots\right)\left[N(1) q^{4}+N(2) q^{8}+N(3) q^{12}+\ldots \ldots\right]
$$

is equal to

$$
\sum_{r} M\left(\frac{m}{r}\right) \lambda(r) \mu(r)
$$

where $\mu(n)$ is the Möbius function, and $N(n)$ and $M(n)$ are the same as those stated in Theorem 0 . We can restate this fact as

$$
2 \sum_{r}^{\prime} N\left(\frac{m^{2}-r^{2}}{4}\right)=\sum_{r} M\left(\frac{m}{r}\right) \lambda(r) \mu(r) .
$$

Now remembering the multiplicative property of $\lambda(x)$ we have

$$
\lambda(r) \cdot \lambda\left(\frac{m}{r}\right)=\lambda(m) \text {. }
$$

In virtue of the above relation and the fact that $\lambda(u) \neq 0$ we obtain from (5) the following

$$
\frac{2}{\lambda(m)} \sum_{r}^{\prime} N\left(\frac{m^{2}-r^{2}}{4}\right)=\sum_{r} \mu(r) M\left(\frac{m}{r}\right) / \lambda\left(\frac{m}{r}\right)=\sum_{r\lceil m} \mu\left(\frac{m}{r}\right) M(r) / \lambda(r) .
$$

Theorem 0 is now easily established from (6) by the use of the Möbius inversion formula [2].

\section{Proof of the theorems}

Remembering Lemmas 1 and 2 and writing successively $\sigma(n), n \sigma(n)$ and $\sigma_{3}(n)$ in place of $N(n)$, and $n, n^{3}$ and $n^{3}$ for $\lambda(n)$ in the basic theorem we can show that 


$$
\begin{aligned}
& \frac{1}{24}\left[5 \sigma_{3}(n)-(\sigma n-1) \sigma(n)\right]=\sum_{d \Gamma_{n}}\left[\frac{1}{d} \sum_{r}^{\prime} \sigma\left(\frac{d^{2}-r^{2}}{4}\right)\right], \\
& \frac{1}{6 n}\left[\sigma_{3}(n)-n \sigma(n)\right]=\sum_{d\rceil n}\left[\frac{1}{d^{3}} \sum_{r}^{\prime}\left(d^{2}-r^{2}\right) \sigma\left(\frac{d^{2}-r^{2}}{4}\right)\right], \\
& \frac{1}{240 n^{3}}\left[\sigma_{7}(n)-\sigma_{3}(n)\right]=\sum_{d \prod_{n}}\left[\frac{1}{d^{3}} \sum_{r}^{\prime} \sigma_{3}\left(\frac{d^{2}-r^{2}}{4}\right)\right] .
\end{aligned}
$$

Theorem 1 follows easily from (7) and (8) by the elimination of $\sigma_{3}(n)$ between them. Theorem 2 is equally simple, - we subtract (8) from (7). Theorem 3 is an obvious consequence of the relation (9).

Again remembering Lemmas 1 and 3 and writing successively $n^{2} \sigma(n)$, $n \sigma_{3}(n)$ and $\sigma_{5}(n)$ in place of $N(n)$, and $n^{5}$ for $\lambda(n)$ in the basic theorem one can obtain without difficulty the following.

$$
\begin{gathered}
\frac{1}{7 n} \cdot \sigma_{3}(n)-\frac{2}{15} \cdot \sigma(n)-\frac{1}{105 n^{5}} \cdot \tau(n)=\sum_{d\lceil n}\left[\frac{1}{d^{5}} \sum_{r}^{\prime}\left(d^{2}-r^{2}\right)^{2} \sigma\left(\frac{d^{2}-r^{2}}{4}\right)\right], \\
\frac{1}{270 n^{3}} \cdot \sigma_{7}(n)-\frac{1}{270 n^{5}} \cdot \tau(n)=\sum_{d \mid n}\left[\frac{1}{d^{5}} \sum_{r}^{\prime}\left(d^{2}-r^{2}\right) \sigma_{3}\left(\frac{d^{2}-r^{2}}{4}\right)\right], \\
65 \sigma_{11}(n)+691 \sigma_{5}(n)-756 \tau(n)=348264 n^{5} \cdot \sum_{d \mid n}\left[\frac{1}{d^{5}} \sum_{r}^{\prime} \sigma_{5}\left(\frac{d^{2}-r^{2}}{4}\right)\right] .
\end{gathered}
$$

The first part of Theorem 4 is obtained easily by subtracting twice the relation ( 8 ) from the sum of the relations (7) and (10). The second part is obtained by subtracting (11) from (9). The last part is a direct consequence of (12).

To prove Theorem 5 we note that in virtue of the relation [1]

$$
\tau\left(p^{\lambda} n\right)=\tau(p) \tau\left(p^{\lambda-1} n\right)-p^{11} \tau\left(p^{\lambda-2} n\right)
$$

one is justified in substituting $\tau(n)=p_{24}(n-1)$ for $N(n)$, and 11 for $\alpha$ in Theorem 0 . The first part of Theorem 5 is an immediate consequence when one notes that

$$
\left[\sum_{1}^{\infty} \tau(n) x^{n}\right]^{2}=x^{2}\left[\prod_{1}^{\infty}\left(1-x^{n}\right)\right]^{48}=\sum_{2}^{\infty} p_{48}(n-2) x^{n} .
$$

The second part of the theorem follows easily on a joint consideration of the first part of Theorem 5 and the last part of Theorem 4. 


\section{References}

[1] G.H. Hardy, Ramanujan (Cambridge University Press, Cambridge, 1940).

[2] G.H. Hardy and E.M. Wright, An introduction to the theory of numbers (Clarendon Press, Oxford, 1938).

[3] Adolf Hurwitz, Mathematische Werke, Band II, (Birkhaüser, Basel, 1933).

[4] D.B. Lahiri, "On Ramanujan's function $\tau(n)$ and the divisor function $\sigma_{k}(n)-I^{\prime \prime}$, Buli. Calcutta Math. Soc. 38 (1946), 193-206.

[5] D.B. Lahiri, "On Remanujan's function $\tau(n)$ and the divisor function $\sigma_{k}(n)-I I^{\prime \prime}$, BuzZ. Calcutta Math. Soc. 39 (1947), 33-52.

[6] D.B. Lahiri, "Identities connecting the partition, divisor and Ramanujan's functions", Proc. Nat. Inst. Sci. India, 34A (1968). 96-103.

[7] 1. Niven and H.S. Zuckerman, An introduction to the theory of numbers, (John Wiley, New York, 1960).

[8] H.F. Sandham, "A square as the sum of 7 squares", Quart. J. Math., Oxford Ser. (2), 4 (1953), 230-236.

[9] H.F. Sandham, "A square as the sum of 9,11 and 13 squares", J. London Math. Soc. 29 (1954), 31-38.

Indian Statistical Institute,

Calcutta, India. 